\title{
DESCOBRINDO PI: DUAS ATIVIDADES BASEADAS NOS TRABALHOS DE ARQUIMEDES
}

\author{
DISCOVERING PI: TWO ACTIVITIES BASED ON THE WORK OF \\ ARCHIMEDES
}

\author{
Lucieli M. Trivizoli ${ }^{1}$
}

Universidade Estadual de Maringá - UEM

\begin{abstract}
Resumo
Neste artigo, oferecemos algumas sugestões para atividades a partir das ideias desenvolvidas por Arquimedes (287-212 a.C.), atividades com raízes na história da Matemática que podem proporcionar uma ligação entre a Matemática de hoje com problemas e matemáticos do passado. Essas atividades envolvem informações sobre Arquimedes e o cálculo do valor aproximado de $\pi$ estão baseadas nas propostas indicadas por Jim Ebert et al., no livro Historical modules for the teaching and learning of mathematics, organizado por Victor J. Katz e Karen Dee Michalowicz (2004). A importância dessas atividades não é de possibilitar o cálculo aproximado de $\pi$ em si, é de, por meio desse cálculo, possibilitar discussões acerca da importância histórica de Arquimedes, da importância do reconhecimento de certos padrões, das relações que se pode fazer com geometria e trigonometria, entre outras.
\end{abstract}

Palavras-chave: Atividades; Informações Históricas; História da Matemática; Arquimedes.

\begin{abstract}
On this paper, some activities for classrooms are suggested involving the ideas developed by Archimedes (ca. 287-212). The activities have roots in the History of Mathematics and they can provide a link between today's Mathematics with problems and mathematicians of the Past. These activities involve information about Archimedes and the approximate Calculation of value of $\pi$, they are based on the schemes indicated by Jim Ebert et al. on the book Historical modules for the teaching and learning of mathematics, organized by Victor J. Katz and Karen Dee Michalowicz (2004). The importance of these activities is not related to enable the approximate calculation of $\pi$ itself, but on the idea of supporting discussions about the historical importance of Archimedes through this calculation, the importance of the recognition of certain patterns, of the relationships it can be made with Geometry and Trigonometry, among others aspects.
\end{abstract}

Keywords: Activities through historical information; History of Mathematics; Archimedes.

\section{Introdução}

\footnotetext{
${ }^{1}$ Endereço eletrônico: Imtrivizoli@uem.br
} 
A História da Matemática além de, obviamente, estar ligada ao conhecimento Matemático, também está conectada à Educação Matemática e à História das Ciências. Os estudos na perspectiva que trata a História da Matemática no ensino de Matemática têm interesse, por exemplo, nas questões de como informações e procedimentos históricos podem ajudar professores e alunos. Miguel e Miorim (2002) destacam nesse ramo os estudos interessados em qualquer tipo de participação da História (da Matemática, da Educação Matemática ou História em um sentido amplo) como parte da educação matemática (ação pedagógica) em diferentes níveis de educação, diversos aspectos ou instituições.

A literatura oferece uma variedade de argumentos para o uso da história em Educação Matemática. Jankvist (2009) destaca que estes argumentos, geralmente, são definidos em duas categorias: a história como uma ferramenta ou a história como um objetivo. Além dos argumentos para a utilização e integração da História da Matemática na Educação Matemática, existem, também, argumentos que questionam seu uso. Essas discussões podem ser encontradas, por exemplo, em Fauvel (1991) e Tzanakis \& Arcavi (2000).

Neste artigo, oferecemos algumas sugestões para atividades a partir das ideias desenvolvidas por Arquimedes (287 a.C. - 212 a.C.). Essas atividades, por sua vez, são baseadas nas propostas indicadas por Ebert et al. (2004) no livro Historical modules for the teaching and learning of mathematics (KATZ, MICHALOWICZ, 2004). A intenção deste artigo é de oferecer uma possibilidade de atividades que possam enriquecer uma aula e/ou auxiliar a colocar o conteúdo de uma aula em um contexto histórico, social e científico. É importante salientar que o professor tem autonomia para incluir essas atividades no momento que achar mais apropriado para a aprendizagem de seus alunos. Ou seja, as atividades podem ser usadas para introduzir um tema, ou para complementar um trabalho que está sendo realizado baseado em livros didáticos ou apostilas, ou ainda como atividades suplementares, uma vez que os alunos tenham uma familiaridade com o assunto.

\section{Sobre Arquimedes e o cálculo do valor aproximado de $\pi$}

Sem dúvida, um dos matemáticos mais famosos de todos os tempos é Arquimedes. Ele viveu durante o século III a.C. em Siracusa, em uma região que atualmente é conhecida como Sicília, na Itália. Arquimedes era bem conhecido por sua 
experiência em questões relacionadas com a matemática, pelos tratados sobre princípios físicos e invenções de máquinas usadas para a guerra (KATZ, 2009)². Embora Arquimedes tenha vivido e morrido em Siracusa, os historiadores assumem que ele tinha comunicação com outros matemáticos da sua época e teria residido algum período no Egito. É provável que Arquimedes passou um tempo de sua juventude em Alexandria, onde pode ter conhecido Eratóstenes ${ }^{3}$.

De acordo com Katz (2009, p.96), Arquimedes foi o primeiro matemático a obter resultados a partir da criação de modelos matemáticos de problemas físicos na terra. Um de seus trabalhos mais conhecidos é sobre a lei da alavanca ${ }^{4}$ e sobre seus cálculos que demonstram que o valor de $\pi$ está entre $3 \frac{10}{71}$ e $3 \frac{10}{70}$. Em uma representação decimal, com valores aproximados, $\pi$ está entre 3,14084507042 e 3,14285714285 .

Esta aproximação para $\pi$ foi obtida a partir de seu conhecimento de que o comprimento de um círculo é proporcional a seu diâmetro $\left(\frac{c}{d}\right)$ e foi apresentada em seu trabalho Medida do Círculo. Arquimedes desenvolveu suas ideias ao tentar encontrar limites superiores e inferiores para esta razão ao circunscrever e inscrever polígonos regulares de $n$ lados a um círculo. Entre muitas realizações originais e impressionantes de Arquimedes, seu método para encontrar uma aproximação numérica para $\pi$ se destaca por ser elegante, fácil de seguir e por fornecer uma boa aproximação para o valor de $\pi$.

\footnotetext{
${ }^{2}$ Uma famosa história diz que o rei tinha encomendado uma coroa de folhas de ouro puro a um ourives. Entretanto, havia um boato de que a coroa havia sido feita com prata. O rei, então, pediu a Arquimedes para provar se a coroa foi, de fato, feita de ouro puro. De acordo com essa história, enquanto Arquimedes estava sentado em uma banheira notou o deslocamento de água. Ele percebeu que objetos com o mesmo peso, mas de densidade diferente, deslocariam diferentes quantidades de água. Assim, ele poderia dizer se a coroa era de puro ouro ou não, por meio da verificação da quantidade de água deslocada. Nessa história, conta-se que ele ficou tão animado por sua descoberta e solução para o problema, que ele deu um pulo, esquecendo as suas vestes, e correu pelas ruas gritando "Eureka, Eureka" (Descobri, descobri). Dessa maneira, Arquimedes havia descoberto que o ourives tinha enganado o rei, através de princípios físicos. A veracidade dessa história é analisada por Martins (2000), onde são analisados diversos aspectos sobre os elementos dessa histórias e indica que não foi esse o método que Arquimedes utilizou. Cf. MARTINS, R. A. Arquimedes e a Coroa do Rei: problemas históricos. Caderno Catarinense de Ensino de Física, v. 17, $\quad$ n. $\quad 2, \quad$ p.115-121, 2000. $\quad$ Disponível em https://periodicos.ufsc.br/index.php/fisica/article/download/6769/6238>. Acesso em 7 de Março de 2016. 3 O livro de Arquimedes intitulado "O Método", dado a conhecer por J. L. Heiberg em 1906, traz uma carta direcionada a Eratóstenes de Alexandria (ÁVILA, 1986).

4 Um livro sobre Arquimedes, o Centro de Gravidade e a Lei da Alavanca está disponível em: <http://www.ifi.unicamp.br/ assis>. Acesso em 07 de Março de 2016.
} 
Baseado neste tema desenvolvido por Arquimedes, este artigo sugere duas atividades para serem desenvolvidas em sala de aula. Apesar de estarem elencadas em uma certa ordem, elas não precisam ser desenvolvidas em sequência. O professor pode utilizar uma (ou as duas) no momento que considerar conveniente no ensino para seus alunos.

\section{Propostas de atividades}

Uma atividade já bastante conhecida entre professores é aquela que utiliza vários materiais circulares para identificar um padrão na relação entre o comprimento da circunferência e seu diâmetro. O professor pode trazer (ou solicitar aos alunos) diversos objetos como tampas de diferentes tamanhos, discos, CDs, latas, pratos etc., além de barbante, régua, calculadora. Os alunos já devem compreender o significado de circunferência, diâmetro, raio para realizar as medições. Um modelo como a Tabela 1 pode ser utilizada pelos estudantes

Tabela 1: registros das medições

\begin{tabular}{|l|l|l|}
\hline Comprimento da circunferência & Comprimento do Diâmetro & $\begin{array}{l}\text { Comprimento da Circunferência } \\
\div \text { comprimento de diâmetro }\end{array}$ \\
\hline & & \\
\hline & & \\
\hline
\end{tabular}

Fonte: Adaptado de Jim Ebert et al. (2004).

Os alunos devem enrolar o barbante no exterior da tampa (ou outro objeto circular), medir o comprimento do barbante, medir o diâmetro da tampa (ou outro objeto circular) e por fim, realizar o cálculo (pode ser feito com a ajuda de uma calculadora) para completar a última coluna da tabela.

Os procedimentos podem ser realizados em grupo ou duplas e então discutidos na classe, buscando identificar as relações entre as medições e os valores da última coluna da tabela 1, tentando identificar alguma similaridade nos valores que vão aparecer na última coluna.

Arquimedes calculou um valor aproximado para $\pi$ há mais de 2.000 anos. Sua aproximação era extremamente precisa e foi insuperável por mais de 1.000 anos. Uma pergunta que sempre pode ser discutida é: "Como é que eles calcularam $\pi$ no passado?" E ainda "Por que assumimos que $\pi$ tem um valor aproximado a 3,14 ? Ou por que pode- 
se usar a aproximação de 22/7 para o valor de $\pi$ ?". Pode-se incluir, também, uma discussão que indique que naquele período não era sabido que $\pi$ é um número irracional e que o símbolo " $\pi$ ” que só começa a ser adotado em 1706 (STEIN, 1999 apud EBERT et al., 2004, p. 13).

A segunda atividade proposta envolve a obtenção de um valor aproximado para $\pi$ utilizando a ideia de Arquimedes que envolvia sucessivas delimitações do comprimento da circunferência por meio do cálculo do perímetro de polígonos regulares inscritos e circunscritos à circunferência. O processo usado por Arquimedes é coerente, mas os cálculos podem ser difíceis e demorados, por isso a sugestão de se utilizar uma calculadora.

Vamos considerar as circunferências de raio $=1$. Arquimedes conhecia a relação de que o comprimento de uma circunferência é proporcional a seu diâmetro. Conhecendo-se o raio $(r)$, sabe-se o diâmetro $(d)$; sabendo-se o comprimento $(c)$ da circunferência é possível conhecer um valor aproximado dessa razão $\left(\frac{c}{d}\right)$. Para uma aproximação do comprimento da circunferência, Arquimedes calculou o perímetro de polígonos regulares inscritos e circunscritos a uma circunferência. Dessa forma, para determinar o perímetro do polígono basta conhecer a medida de um de seus lados. Note que (figura 1) conforme o número de lados do polígono regular aumenta, mais próximo seu perímetro fica do comprimento da circunferência.

Figura 1: Polígonos regulares inscritos numa circunferência de raio 1.
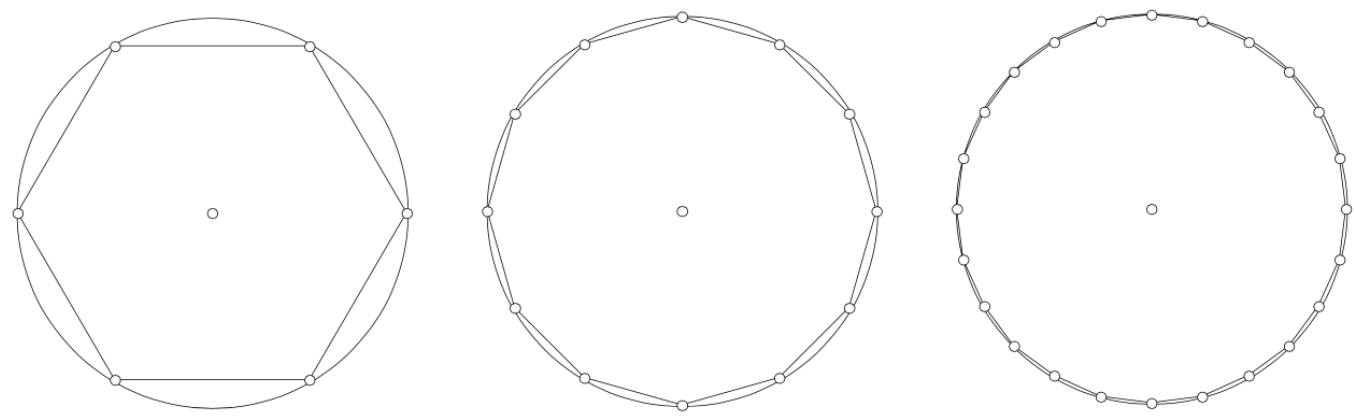

Fonte: A autora.

Podemos iniciar comentando os casos de polígonos regulares inscritos. Começamos com o hexágono regular e a partir dele podemos encontrar a medida dos lados dos polígonos de 12 lados, 24 lados, 48 lados e 96 lados. Baseados na técnica de 
Arquimedes, vamos encontrar a medida dos lados desses polígonos. Os elementos geométricos para esses cálculos são observados na figura a seguir:

Figura 2: Hexágono regular inscrito na circunferência de raio $=1$.

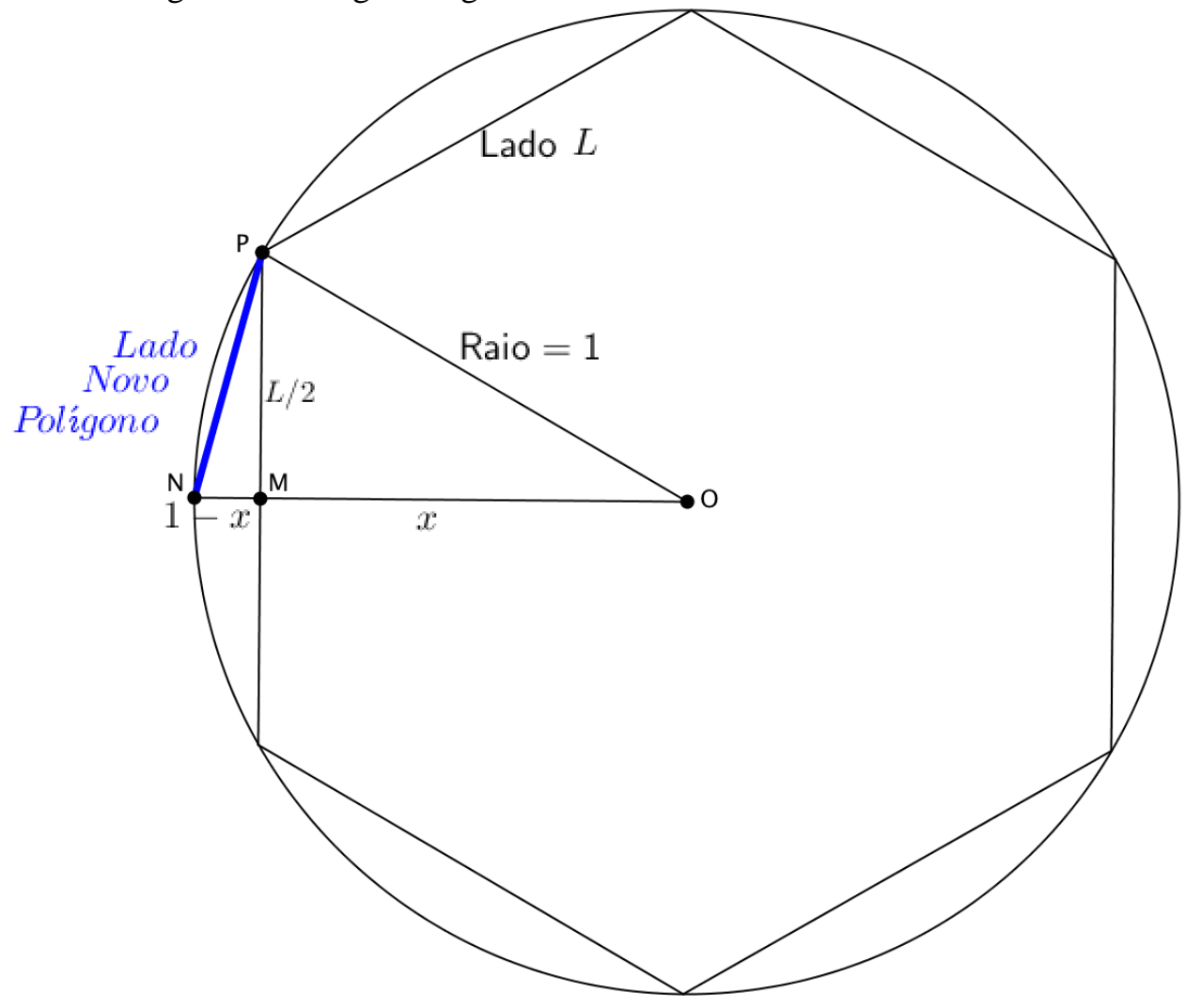

Fonte: Adaptado de Jim Ebert et al. (2004).

Ao traçar um raio $(\mathrm{ON})$ pelo ponto médio $(\mathrm{M})$ de um lado do polígono regular, divide-se o lado e o arco correspondente a este lado pela metade. $\mathrm{O}$ ponto médio $(\mathrm{N})$ do arco e o vértice $(\mathrm{P})$ do lado do polígono original formam o seguimento que será o novo lado para polígono de 12 lados. Podemos utilizar-se das propriedades geométricas de polígonos regulares e circunferências, e do Teorema de Pitágoras (nos triângulos OMP e PMN) para encontrar o comprimento do novo lado. Em seguida, repetindo todo o processo e com a ajuda de uma calculadora, consegue-se determinar o comprimento dos lados dos polígonos de 24, 48 e 96 lados.

Procedimentos parecidos podem ser seguidos para fazer os cálculos dos perímetros dos polígonos circunscritos à circunferência e um modelo, como a tabela 2 , pode ajudar na orientação e organização das informações. 
Tabela 2: registros dos cálculos

\begin{tabular}{|c|c|c|c|c|}
\hline № de Lados & Comprimento do Lado Perímetro (P) & Diâmetro (D) & P / D \\
\hline 6 & & & 2 & \\
\hline 12 & & & 2 & \\
\hline 24 & & & 2 & \\
\hline 48 & & & 2 & \\
\hline 96 & & & 2 & \\
\hline
\end{tabular}

Fonte: Adaptado de Jim Ebert et al. (2004).

A análise dos valores da última coluna deve ainda ser direcionada para a relação entre o comprimento da circunferência e seu diâmetro. Calculando-se o perímetro de polígonos regulares inscritos e circunscritos a uma circunferência e relacionando esses perímetros com o diâmetro dessa circunferência, calculam-se boas aproximações para $\pi$, a menor e a maior.

\section{Considerações}

A importância dessas atividades não é de possibilitar o cálculo aproximado de $\pi$ em si, é de, por meio desse cálculo, possibilitar discussões acerca da importância histórica de Arquimedes, da importância do reconhecimento de certos padrões, das relações que se pode fazer com geometria e trigonometria, entre outras. Essas atividades podem ajudar os alunos a estabelecer conexões entre a forma e a área de polígonos, entre iteração e estimativa, a reconhecer e usar relações entre as ideias matemáticas. Além disso, essas atividades têm suas raízes na história, e podem proporcionar uma ligação entre a matemática de hoje com problemas e matemáticos do passado.

\section{Referências}

ÁVILA, Geraldo. Arquimedes, a esfera e o cilindro. RPM 10, 1986.Disponível em <http://www.rpm.org.br/cdrpm/10/3.htm>. Acesso em 13 de Novembro de 2017. 
Washington, D.c.: Mathematical Association Of America, 2004. Cap. 1. p. 1-118. (Historical Modules Project). CD-ROM.

FAUVEL, John. Using History in Mathematics Education. For The Learning Of Mathematics, Edmonton, v. 11, n. 2, p.3-6, jun. 1991. Special Issue on History in Mathematics Education. Disponível em: 〈http://www.jstor.org/stable/40248010>. Acesso em: 29 jul. 2015.

JANKVIST, Uffe Thomas. A categorization of the "whys" and "hows" of using history in mathematics education. Educational Studies In Mathematics, [s.1.], v. 71, n. 3, p.235-261, 21 jan. 2009.

KATZ, Victor J.. A History of Mathematics: An Introduction. 3. ed. Boston: Pearson Education, 2009.

MARTINS, Roberto de Andrade. Arquimedes e a Coroa do Rei: problemas históricos. Caderno Catarinense de Ensino de Física, v. 17, n. 2, p.115-121, 2000. Disponível em < https://periodicos.ufsc.br/index.php/fisica/article/download/6769/6238>. Acesso em 7 de Março de 2016.

MIGUEL, Antonio; MIORIM, Maria Ângela. História da Matemática: uma prática social de investigação em construção. Educação em Revista, Faculdade de Educação da Universidade Federal de Minas Gerais, n. 36, p.177-203, dez. 2002.

TZANAKIS, Constantinos; ARCAVI, Abraham. Integrating history of mathematics in the classroom: analytic survey. In: FAUVEL, John; VAN MAANEN, Jan (Ed.).

History in Mathematics Education: The ICMI Study. Netherlands: Springer, 2002. p. 201-240 